\title{
On New Gauge Boson Signals According to the Littlest Higgs Model in Future $e^{+} e^{-}$Colliders
}

\author{
F. M. L. de Almeida Jr., Y. A. Coutinho, J. A. Martins Simões, A. J. Ramalho, S. Wulck, \\ Instituto de Física, \\ Universidade Federal do Rio de Janeiro, \\ 21941-972, Rio de Janeiro, RJ, Brazil
}

and M. A. B. do Vale

Departamento de Ciências Naturais, Universidade Federal de São João Del Rei, 36301-160, São João del Rei, MG, Brazil

Received on 19 January, 2007

\begin{abstract}
There is a recent proposal of identifying the Higgs particle of the Standard Model as a pseudo NambuGoldstone boson. This new broken symmetry introduces new particles and new interactions. Among these new interactions a central role to get an experimental signal of a new physics is played by the new neutral gauge bosons, $A_{H}$ and $Z_{H}$. We study the associated production of general new neutral gauge boson $Z^{\prime}$ and a hard photon in the process $e^{+}+e^{-} \longrightarrow \mu^{+}+\mu^{-}+\gamma$. For $M_{A_{H}}<\sqrt{s}$ we show that the hard photon energy distribution in $e^{+}+e^{-} \longrightarrow \gamma+f+\bar{f}$ can present a model dependence and establish the theoretical origin of a new possible heavy neutral gauge boson.
\end{abstract}

Keywords: Littlest Higgs; New Gauge Bosons

\section{THE LITTLEST HIGGS MODEL}

Recently, new models have been proposed as possible solutions to the hierarchy problem of the Standard Model (SM). The Little Higgs models [1] implement the idea that the Higgs boson is a pseudo-Goldstone boson. They are constructed by embedding the Standard Model inside a larger group with an enlarged symmetry. The minimal model was called the Littlest Higgs Model (LHM). The LHM consists of an SU(5) non-linear sigma model which is spontaneously broken to its subgroup $S O(5)$ by vacuum expectation value (VEV) of order $f$. The gauged group $(S U(2) \otimes U(1))^{2}$ is broken at the same time to its diagonal electroweak SM subgroup $S U(2) \otimes U(1)$. The new heavy states in this model consists of heavy gauge bosons $\left(W_{H}, Z_{H}, A_{H}\right)$, a triplet Higgs $\Phi$ and a vector like top quark which cancels the quadratic divergences coming from the SM top quark. By construction, all of the new states acquire masses of order $f$ and typically in the TeV range. The approximate mass relations for the heavy gauge bosons can be written as [2]:

$$
M_{W_{H}}^{2} \simeq M_{Z_{H}}^{2} \geq \frac{M_{W}^{2} 4 f^{2}}{v^{2}} \quad M_{A_{H}}^{2} \geq M_{w}^{2} \tan ^{2} \theta_{w} \frac{4 f^{2}}{5 v^{2}}
$$

where $\theta_{w}$ is the Weinberg angle, $v$ is the electroweak scale and the mass parameter $M_{W} \equiv g v / 2$ approaches the SM $W$-boson mass when $f \rightarrow \infty$. The neutral gauge boson $A_{H}$ is typically light and should be the first signal of the LHM. A first property of this model is the presence of a second, heavier, new neutral gauge boson, named $Z_{H}\left(M_{Z_{H}} \approx 4 M_{A_{H}}\right)$. The total decay width $\Gamma_{Z_{H}}$ is mainly dependent of the free parameters $M_{Z_{H}}$ and $c(\cos \theta)$, while the total decay width $\Gamma_{A_{H}}$ is sensitive to the free parameters $M_{A_{H}}$ and $c^{\prime}\left(\cos \theta^{\prime}\right)$. The parameter space for the two mixing angles, $c$ and $c^{\prime}$, is $0 \leq c \leq 0.5$ and $0.62 \leq c^{\prime} \leq 0.73$, consistent with the precision electroweak constraints.

\section{THE HARD PHOTON}

The most direct channel to study the $A_{H}, Z_{H}$ properties is $e^{+}+e^{-} \longrightarrow f+\bar{f}$, at the ressonant energy $\sqrt{s}=M_{Z_{H}}, M_{A_{H}}$. As these masses are unknown, the new high-energy accelerators will have to vary $\sqrt{s}$. As this procedure is a very complex operation it is important to have physical alternatives at fixed $\sqrt{s}$.

In this work we explore the interesting properties of the fundamental interaction mechanism of the associated production of new neutral gauge bosons and a hard photon in the process $e^{+}+e^{-} \longrightarrow \mu^{+}+\mu^{-}+\gamma$. (For more details see [3]). The Feynman diagrams for this channel are shown in Fig. 1. A

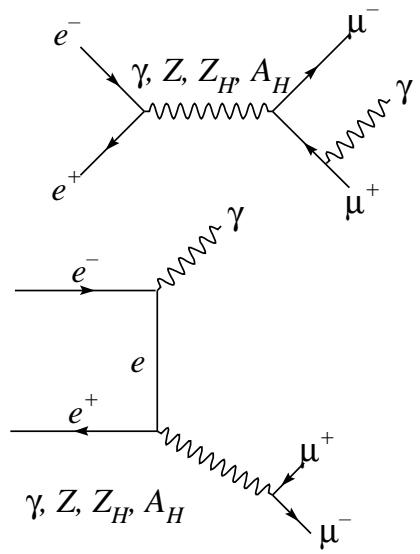

FIG. 1: Feynman diagrams for the process $e^{+}+e^{-} \longrightarrow \mu^{+}+\mu^{-}+\gamma$.

very simple consequence of the conservation of energy and momentum is that the final high energy photon has a fixed 


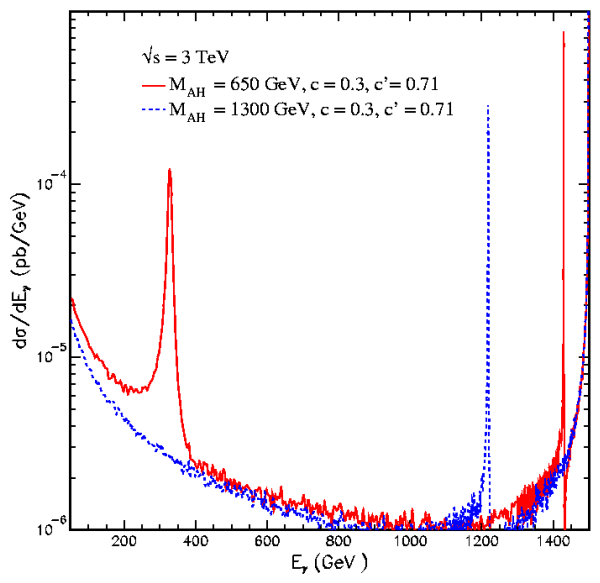

FIG. 2: Photon energy distribution in $e^{+}+e^{-} \longrightarrow \gamma+\mu^{-}+\mu^{+}$showing the two peaks associated to $Z_{H}$ and $A_{H}$ with $\sqrt{s}=3 \mathrm{TeV}$ for LHM $\left(c=0.3\right.$ and $\left.c^{\prime}=0.71\right)$.

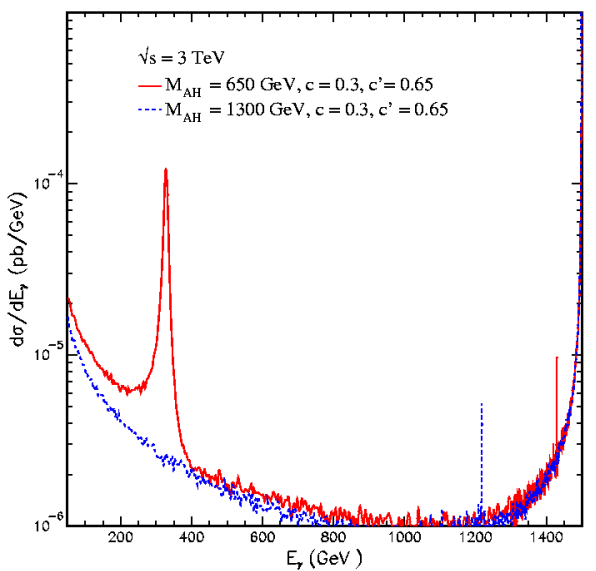

FIG. 3: Photon energy distribution in $e^{+}+e^{-} \longrightarrow \gamma+\mu^{-}+\mu^{+}$showing the two peaks associated to $Z_{H}$ and $A_{H}$ with $\sqrt{s}=3 \mathrm{TeV}$ for LHM $\left(c=0.3\right.$ and $\left.c^{\prime}=0.65\right)$.

energy given by [4].

$$
E_{\gamma} \mp \Delta_{\gamma}=\frac{s-\left(M_{Z^{\prime}} \pm \Delta_{Z^{\prime}}\right)^{2}}{2 \sqrt{s}}
$$

where $\Delta_{\gamma}$ and $\Delta_{Z^{\prime}}$ are the uncertainties in the photon energy and in $M_{Z^{\prime}}$.

The study of the hard photon energy distribution will give most information as the direct new bosons decays, but in a simple and direct way. Since the new boson masses are not known, colliders are considered at the highest energy and so we are considering $\sqrt{s}=3 \mathrm{TeV}$. If indications of new bosons are found with masses lower than $\sqrt{s}$, a new run with a c.m. energy near the mass value is not necessary in order to study the new bosons properties in details.

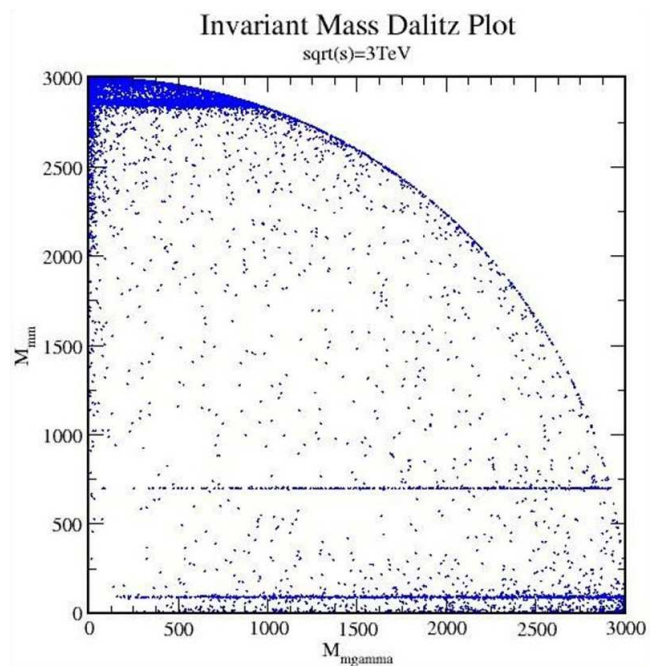

FIG. 4: Dalitz Plot.

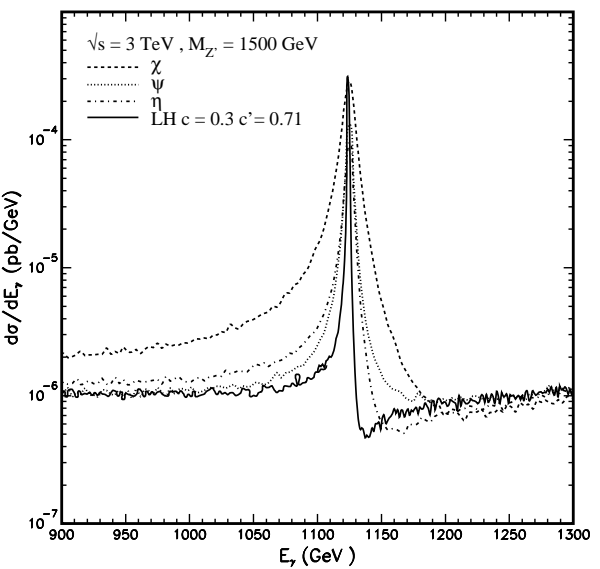

FIG. 5: Photon energy distribution in $e^{+}+e^{-} \longrightarrow \gamma+\mu^{-}+\mu^{+}$with $\sqrt{s}=3 \mathrm{TeV}$ for $\chi, \eta, \psi$ and for LHM $\left(c=0.3\right.$ and $\left.c^{\prime}=0.71\right)$.

\section{RESULTS}

In order to obtain the hard photon energy distribution, we imposed a set of realistic cuts: all final states particles were required to emerge with a polar $\cos \theta$, measured with respect to the direction of the electron beam, in the range $|\cos \theta| \leq$ 0.995 . The hard photon energy was required to be greater than $5 \mathrm{GeV}$. The resulting photon energy distributions are shown in Figs. 2 and 3 in the channel $\mu^{+} \mu^{-} \gamma$ for two different $c^{\prime}$ values $\left(c^{\prime}=0.65\right.$ and $\left.c^{\prime}=0.71\right)$. For $M_{A_{H}}=650 \mathrm{GeV}$, we can see two peaks, the first one associated to $Z_{H}$, and the second to $A_{H}$. For $M_{A_{H}}=1300 \mathrm{GeV}$, only the peak associated to $A_{H}$ appears. We remark the strong mixing angle dependence on the magnitude 
of the photon peak. We show in Fig. 4 the invariant mass Dalitz plot. The three horizontal lines correspond to the three different neutral gauge boson $\left(Z, A_{H}, Z_{H}\right)$ masses. Depending on the heavy boson masses one can see two horizontal lines ( $Z$ and $A_{H}$ ) or even only the SM $Z$ mass. In Fig. 5 we can see the hard-photon energy distribution associated with the $A_{H}$ compared to the canonical $\eta, \chi, \psi$ super-string inspired $E_{6}$ models. The $\eta, \chi, \psi$ models have a much larger value for the total neutral gauge boson width and this fact makes the hard photon energy much broader.

\section{CONCLUSIONS}

We have shown that the hard photon energy distribution can establish the theoretical origin of a new possible heavy neutral gauge boson independent of how all new boson decays. This alternative signature for $Z^{\prime}$ production at the new electron-positron colliders could allow us to study its properties, at a fixed collider energy $\sqrt{s}>M_{Z^{\prime}}$. Moreover, if the neutral gauge boson is identified, due to the width of the hard photon energy distribution, as the $A_{H}$ of the LHM, this would imply the existence of another neutral gauge boson, the $Z_{H}$, with mass approximately four times the $A_{H}$ mass.

Acknowledgments: CNPq, FAPERJ and FAPEMIG.

[1] N. Arkani-Hamed, A. G. Cohen, and H. Georgi, Phys. Lett. B 513, 232 (2001) [arXiv:hep-ph/0105239].

[2] T. Han, H. E. Logan, B. McElrath, and L. T. Wang, Phys. Rev. D 67, 095004 (2003) [ar-Xiv:hep-ph/0301040].

[3] F. M. L. Almeida Jr., Y. A. Coutinho, J. A. Martins Simões, A. J. Ramalho, S. Wulck, and M. A. B. do Vale, [arXiv:hep-

$\mathrm{ph} / 0601102]$.

[4] F. M. L. Almeida Jr., Y. A. Coutinho, J. A. Martins Simões, A. J. Ramalho, S. Wulck, and M. A. B. do Vale, Eur. Phys. J. C 44, 127 (2005). 